\title{
Digenean parasites of the great antshrike, Taraba major (Aves: Thamnophilidae), from Argentina, with a description of a new species of the genus Strigea (Strigeidae)
}

\author{
Lía I. Lunaschi and Fabiana B. Drago
}

División Zoología Invertebrados, Museo de La Plata, Facultad de Ciencias Naturales y Museo, La Plata, Buenos Aires, Argentina

\begin{abstract}
During a survey of birds from Argentina, two species of Digenea, one of them new, were found parasitizing the great antshrike, Taraba major (Vieillot) (Aves: Thamnophilidae). The strigeid, Strigea orbiculata sp. $\mathrm{n}$. is characterized by having a body plump, a copulatory bursa without a membraneous fold ('Ringnapf'), entire testes, eggs with miracidia with eye-spots, by the arrangement of vitelline follicles in the forebody, which are densely distributed from its anterior edge, and by the absence of a neck region in the hindbody. Among the known Neotropical species of Strigea Abildgaard, 1790, only five share with Strigea orbiculata sp. n. the body shape and the distribution of vitelline follicles in the forebody: Strigea caluri Dubois, 1962, S. elliptica (Brandes, 1888), S. inflecta Lunaschi et Drago, 2012, S. nugax Szidat, 1928 and S. sphaerocephala (Westrumb, 1823 nec Brandes 1888). However, $S$. caluri can be easily distinguished by having a membraneous fold in the copulatory bursa originated from 'Ringnapf', and multilobed testes. Strigea elliptica differs mainly by having a well developed 'Ringnapf' and the remaining species differ principally by metrical characters. The dicrocoeliid, Lyperosomum oswaldoi (Travassos, 1919) is reported for the first time from Argentina and T. major represents its new definitive host. The host specificity of Neotropical Strigea spp. is discussed and an updated list of records of their hosts is provided.
\end{abstract}

Keywords: Strigea orbiculata sp. n., Dicrocoeliidae, Lyperosomum oswaldoi, Passeriformes, South America, Digenea, taxonomy, birds

The great antshrike, Taraba major (Vieillot), is a bird of wide distribution in the Neotropical region: northern and eastern Bolivia, southcentral Brazil, western Paraguay and northern Argentina. Its diet is mainly composed of insects and other arthropods, snails and other molluscs, but also of small vertebrates, including lizards, frogs, tadpoles, minnows and small mammals, and vegetable matter (Zimmer and Isler 2003). The helminth fauna of the great antshrike is poorly known, with only two species, Prosthogonimus ovatus (Rudolphi, 1803) (Digenea: Prosthogonimidae) and Diplotriaena sp. (Nematoda: Diplotriaenidae), reported from Brazil (Kohn and Fernandes 1972, Vicente et al. 1982).

During a helminthological study of birds from Formosa Province, Argentina, two digenean species were recovered from the small intestine and the bile duct of T. major. Examination of this material revealed the presence of one new species of Strigea Abildgaard, 1790 (Strigeidae), and one known species of Lyperosomum Looss, 1899 (Dicrocoeliidae). Both species are described in the present paper.

\section{MATERIALS AND METHODS}

Seven specimens of Taraba major were collected in September 2009 and June and September 2012 from La Marcela farm
(26 $\left.17^{\circ} 35^{\prime \prime} \mathrm{S}, 5^{\circ} 08^{\prime} 38^{\prime \prime} \mathrm{W}\right)$, Pirané, Formosa Province, Argentina. The birds were collected with authorization of the Ministerio de la Producción y Ambiente of Formosa Province, dissected in the field and their viscera were analysed immediately after capture. The digeneans were removed, fixed in $5 \%$ formalin, stained with hydrochloric carmine (1: 6 dilution in $96 \%$ ethanol), dehydrated, cleared in xylene or beechwood creosote and mounted in Canada balsam. The drawings were made with the aid of a drawing tube. Measurements are given in micrometres $(\mu \mathrm{m})$ unless otherwise stated, as the range followed by the mean in parentheses. The helminths were deposited at the helminthological collection of the Museo de La Plata (MLP) and the birds were deposited at the ornithological collection of the Museo de La Plata, in La Plata, Argentina. The terms oioxenous and euryxenous utilized were used according to Combes (2001).

\section{RESULTS}

Strigeidae Railliet, 1919

Strigea Abildgaard, 1790

Strigea orbiculata sp. n.

Figs. 1-2, Tables 1-2

Description (based on six gravid specimens): Body plump, 0.96-1.21 mm (1.04 mm) in total length. Tegumental spines only on anteroventral surface of forebody. 
Forebody wider than long, hemispherical, almost truncated anteriorly, with a large opening covered with minute delicate spines, 348-580 (433) long by 580-752 (622) wide. Hindbody cylindrical, strongly curved dorsally, with its posterior end close to forebody, almost two times longer than forebody, 546-774 (645) long by 386-638 (462) wide. Ratio of hindbody length to forebody length 1.3-1.8 (1.6).

Oral sucker subterminal, well developed, 97-121 (109) long by 101-145 (122) wide. Ventral sucker well developed, larger than oral sucker, 145-174 (159) long by 155-184 (174) wide. Sucker-width ratio 1.3-1.5 (1.4). Holdfast organ lobes can be projected beyond anterior margin of forebody; proteolytic gland at base of forebody, difficult to see in some individuals, 55-83 (69) long by 107-134 (121) wide. Prepharynx absent; pharynx 71-107 (93) long by 41-71 (54) wide; oesophagus and intestinal caeca not discernible. Ratio of pharynx length to oral sucker length 0.6-1.1 (0.8).

Testes in tandem, situated near posterior end of body, rounded and slightly overlapping; anterior testis 110-190 (141) long by 238-369 (287) wide; posterior testis 119-186 (157) long by 181-381 (244) wide. Seminal vesicle long, folded on itself.

Ovary ovoid, 46-83 (69) long by 102- 143 (125) wide, at distance of 238-274 (250) from intersegmental constriction. Laurer's canal not seen. Mehlis' gland and vitelline reservoir in intertesticular region. Vitelline follicles similar in size in both body segments, in forebody extensively distributed in dorsal and ventral walls from anterior end, masking pharynx and ventral sucker, and filling most of ventral lobe and base of dorsal lobe of holdfast organ; in hindbody occupying preovarian region and extending across testicular region to near posterior end.

Uterus with large and numerous eggs, extending up to intersegmental constriction. Eggs operculate, 100-130 long by $71-88$ wide $(116 \times 77)$, containing well-developed miracidia with eye-spots. Ratio of body length to egg length 8-11 (10). Ratio of hindbody length to egg length 5-7 (6).

Copulatory bursa quadrilobed, well delimited, 143-190 (163) long by 202-217 (207) wide; genital atrium shallow, 52-83 (70) in depth; genital pore terminal. Muscular ring ('Ringnapf') absent. Genital cone small, with a truncated base, slender when protruded, 95-136 (113) long by 52-71 (59) wide; ejaculatory duct and uterus join at base of genital cone, forming hermaphroditic duct. Ratio of hindbody length to genital cone length 5-7 (6). Ratio of genital cone length to egg length 0.9-1.2 (1). Excretory vesicle and excretory pore not seen.

Type host: Taraba major (Vieillot) (chororó, great antshrike) (Passeriformes: Thamnophilidae).

Type locality: La Marcela farm, Pirané (26²17'35"S;

590' $\left.38^{\prime \prime} \mathrm{W}\right)$, Formosa Province, Argentina.

Date of collection: September 2009.

Site of infection: Small intestine.
Prevalence: $14 \%$ ( 1 of 7$)$

Intensity of infection: 8 .

Specimens deposited: Holotype MLP 6672; 5 paratypes MLP 6673.

E t y m o log y: The specific name refers to the rounded-shaped body.

Remarks. Among Neotropical species of Strigea, only five species, Strigea caluri Dubois, 1962, Strigea nugax Szidat, 1928, Strigea sphaerocephala (Westrumb, 1823), Strigea elliptica (Brandes, 1888) and Strigea inflecta Lunaschi et Drago, 2012, can be compared with Strigea orbiculata $\mathrm{sp}$. n. by having a body plump and vitelline glands extensively distributed throughout the forebody. However, S. caluri can be easily distinguished from the new species by having a membraneous fold on the copulatory bursa originated from a muscular ring ('Ringnapf'), and multilobed testes. S. nugax can be differentiated from the new species by having vitelline glands not reaching the anterior edge of the forebody, larger body (length of 5.5-6.0 $\mathrm{mm}$ vs $0.96-1.2 \mathrm{~mm}$ ), smaller eggs $(90-100 \mu \mathrm{m} \times 55 \mu \mathrm{m} v s 100-130 \mu \mathrm{m} \times 71-88 \mu \mathrm{m})$, and a higher ratio body length to egg length (60-67 vs 8-11). Strigea sphaerocephala was poorly described, but it can be distinguished from the new species by having a larger body ( $3 \mathrm{~mm}$ vs $0.96-1.2 \mathrm{~mm})$, a wider forebody $(1.0 \mathrm{~mm}$ vs $348-580 \mu \mathrm{m})$, and smaller eggs $(100 \mu \mathrm{m} \times 50 \mu \mathrm{m} v s$ $100-130 \mu \mathrm{m} \times 71-88 \mu \mathrm{m})$. Strigea elliptica differs from $S$. orbiculata by possessing vitelline follicles of different size in both body regions and a well developed muscular ring ('Ringnapf') in the copulatory bursa. Moreover, S. orbiculata has larger eggs $(100-130 \mu \mathrm{m} \times 71-88 \mu \mathrm{m}$ vs 75-101 $\mu \mathrm{m} \times 48-60 \mu \mathrm{m})$, smaller ratio body length to eggs length (8-11 vs 13-20) and the copulatory bursa with a shallow genital atrium (52-83 $\mu \mathrm{m} v s$ 90-143 $\mu \mathrm{m})$. Strigea inflecta differs from the new species in most metrical characteristics, i.e. a larger size of the body, suckers, pharynx, ovary, genital cone, copulatory bursa and smaller eggs (see Table 1).

Three species of Strigea described in other biogeographic regions are similar to the new species by having a plump body, vitelline glands occupying most of the forebody and testes not lobed: S. baylisi Dubois, 1937 and S. nicolli Dubois, 1937 from passeriform, charadriiform and threskiornithid birds in Australia, and S. intermedia Szidat, 1932 described from Corvus albus Müller (Passeriformes, Corvidae) in French Guinea (now Guinea). Nevertheless, $S$. baylisi differs from $S$. orbiculata by having a well-developed muscular ring ('Ringnapf'), a forebody with a small opening, the vitelline glands distributed only in the dorsal wall of the forebody and by some morphometric characters, such as the larger size of the ovary and genital cone, smaller size of eggs and a deeper genital atrium.

Strigea nicolli can be readily differentiated from the new species by the distribution of vitelline glands that are 

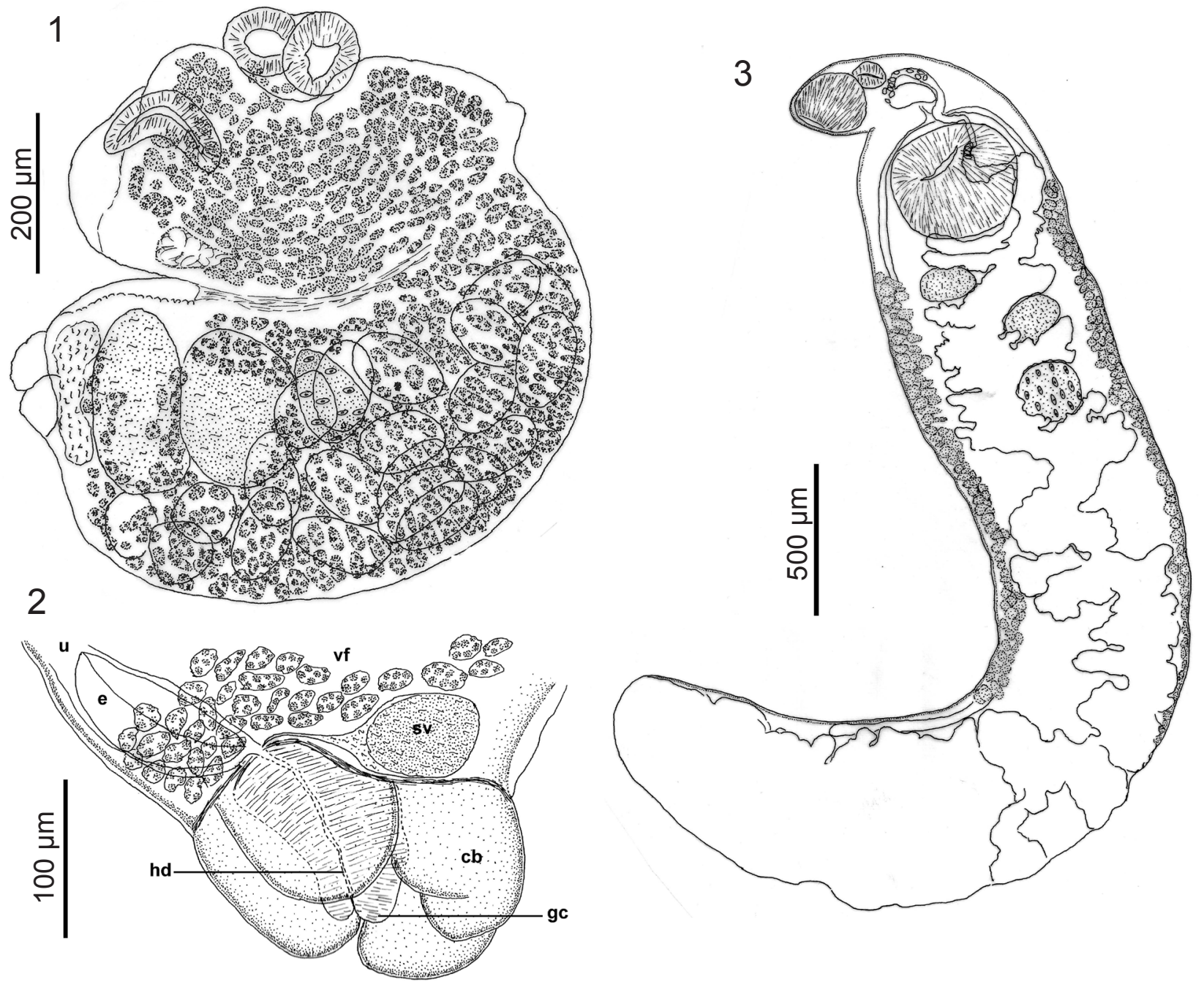

Figs. 1-3. Digeneans from Taraba major. Figs. 1, 2. Strigea orbiculata sp. n. Fig. 1. Entire worm. Fig. 2. Enlarged view of terminal genitalia. Fig. 3. Lyperosomum oswaldoi (Travassos, 1919), entire worm, ventral view. Abbreviations: cb - copulatory bursa; e - egg; $\mathrm{gc}$ - genital cone; hd - hermaphroditic duct; sv - seminal vesicle; $\mathrm{u}$ - uterus; $\mathrm{vf}$ - vitelline follicles.

present only at the dorsal wall of the forebody and extend in the ventral region of hindbody up to its posterior end. Moreover, it differs by having a larger ovary and genital cone. Strigea intermedia can be distinguished from S. orbiculata by having larger dimensions of the body, suckers and pharynx, and smaller eggs (see Table 2). Finally, the presence of eggs in the uterus containing well-developed miracidia with eye-spots is mentioned for the first time in Strigea.

Dicrocoeliidae Looss, 1899

\section{Lyperosomum Looss, 1899}

Lyperosomum oswaldoi (Travassos, 1919) Travassos, 1944

Fig. 3

Description (based on one gravid specimen): Body elongate, wider in post-acetabular region, $4.3 \mathrm{~mm}$ long by $0.68 \mathrm{~mm}$ wide. Ratio of hindbody length to forebody length 7.5. Tegument thin, smooth, sensory papillae not seen. Oral sucker subterminal, round, 238 long by 217 wide. Ventral sucker well developed, larger than oral sucker, situated in end of first quarter of body, 386 long by 430 wide. Suckers width ratio 2 . Prepharynx absent; pharynx oval, 88 long by 114 wide. Oesophagus approximately 131 long; caeca straight, terminated at substantial distance from posterior extremity of body.

Testes located in second body fourth, smooth, transversely oval, oblique in position. Anterior testis located dextral, slightly separated from ventral sucker by loops of uterus, 119 long by 179 wide; posterior testis 133 long by 190 wide. Cirrus sac oval, 143 long by 53 wide, anterior to ventral sucker, containing coiled seminal vesicle. Genital pore median, ventral, postpharyngeal.

Ovary round, with smooth margins, 193 long by 226 wide, situated on same side of body as posterior testis but separated from it by loops of uterus. Mehlis' gland and seminal receptacle postovarian; Laurer's canal not 
Table 1. Comparative data for Strigea orbiculata sp. n. and related species ${ }^{\mathrm{a}}$ from the Neotropical region.

\begin{tabular}{|c|c|c|c|c|c|c|c|}
\hline Species & S. orbiculata sp. n. & S. caluri & S. nugax & $\begin{array}{l}\text { S. sphaero- } \\
\text { cephala }\end{array}$ & S. elliptica & & S. inflecta \\
\hline Locality & Argentina & Central America & Brazil & Brazil & Brazil & Argentina & Argentina \\
\hline Source & Present study & $\begin{array}{l}\text { Dubois } \\
(1962)\end{array}$ & $\begin{array}{l}\text { Dubois } \\
(1938,1968)\end{array}$ & $\begin{array}{l}\text { Dubois } \\
(1938,1968)\end{array}$ & $\begin{array}{l}\text { Dubois } \\
(1938)\end{array}$ & $\begin{array}{l}\text { Lunaschi and Drago } \\
(2009)\end{array}$ & $\begin{array}{l}\text { Lunaschi and Drago } \\
(2012)\end{array}$ \\
\hline $\mathrm{H}$ & $\begin{array}{l}\text { Taraba major } \\
\text { (Vieillot) }\end{array}$ & Pharomachrus mocinno & $\begin{array}{l}\text { Mycteria } \\
\text { americana* }\end{array}$ & $\begin{array}{l}\text { Pyroderus } \\
\text { scutatus } \\
\text { Psarocolius } \\
\text { decumanus }\end{array}$ & $\begin{array}{l}\text { Bubo } \\
\text { virginianus } \\
\text { nacurutu }\end{array}$ & $\begin{array}{l}\text { Buteogallus } \\
\text { meridionalis }\end{array}$ & Cariama cristata \\
\hline B & $0.96-1.2 \mathrm{~mm}$ & $<2.8 \mathrm{~mm}$ & $5.5-6 \mathrm{~mm}$ & to $3 \mathrm{~mm}$ & approx. $2 \mathrm{~mm}$ & $1.14-2.1 \mathrm{~mm}$ & $1.60-1.98 \mathrm{~mm}$ \\
\hline Fo & $348-580 \times 580-752$ & $880-1100 \times 850-1130$ & - & $-\times 1000$ & $600 \times 500$ & $384-613 \times 353-473$ & $561-754 \times 783-1006$ \\
\hline $\mathrm{Hi}$ & $546-774 \times 386-638$ & $1250-1800 \times 850-1240$ & $-\times 1500$ & - & - & $702-1488 \times 324-522$ & $967-1257 \times 532-841$ \\
\hline Os & $97-121 \times 101-145$ & $180-240 \times 160-200$ & mean 250 & mean 100 & mean 100 & $69-106 \times 66-105$ & $183-241 \times 126-155$ \\
\hline Vs & $145-174 \times 155-184$ & $260-290 \times 290-310$ & mean 300 & - & $170-200$ & $102-130 \times 76-115$ & $179-290 \times 174-256$ \\
\hline Pg & $55-83 \times 107-134$ & & - & - & - & $55-95 \times 121-166$ & $72-121 \times 121-174$ \\
\hline $\mathrm{Ph}$ & $71-107 \times 41-71$ & $160-180 \times 130-150$ & mean 150 & mean 60 & mean 50 & $72-87 \times 64-86$ & $140-169 \times 109-126$ \\
\hline $\mathrm{O}$ & $46-83 \times 102-143$ & $180-230 \times 220-320$ & $200-350$ & - & - & $60-97 \times 116-213$ & $111-237 \times 155-222$ \\
\hline At & $110-190 \times 238-369$ & $420-500 \times 700-860$ & $600-700$ & - & $300 \times 400$ & $119-168 \times 190-275$ & $135-232 \times 401-483$ \\
\hline $\mathrm{Pt}$ & $119-186 \times 181-381$ & $480-600 \times 750-860$ & & - & & $167-367 \times 226-290$ & $150-193 \times 338-483$ \\
\hline $\mathrm{Cb}$ & $143-190 \times 202-217$ & - & $800-1200$ & - & - & - & $314-435 \times 483-652$ \\
\hline $\mathrm{Ga}$ & $52-83$ & - & - & - & - & $90-143$ & $232-290$ \\
\hline Gc & $95-136 \times 52-71$ & - & - & - & - & $131-250 \times 48-95$ & $372-435 \times 203-319$ \\
\hline $\mathrm{E}$ & $100-130 \times 71-88$ & $84-99 \times 52-63$ & $90-100 \times 55$ & $100 \times 50$ & $75-100 \times 50-55$ & $81-101 \times 48-60$ & $86-111 \times 50-58$ \\
\hline $\mathrm{Hi} / \mathrm{Fo}$ & $1.3-1.8$ & $1.4-1.8$ & approx. 2 & - & - & $1.3-2.5$ & $1.5-2.1$ \\
\hline $\mathrm{Vs} / \mathrm{Os}$ & $1.3-1.5$ & $1.6-1.8 * *$ & $1.2 * *$ & - & $1.7-2 * *$ & $0.8-1.3$ & $1.4-1.7$ \\
\hline $\mathrm{Ph} / \mathrm{Os}$ & $0.6-1.1$ & $0.8 * *$ & $0.6^{* *}$ & $0.6 * *$ & $0.5 * *$ & $0.8-1.1$ & $0.7-0.8$ \\
\hline $\mathrm{B} / \mathrm{E}$ & 8-11 & $22-33 * *$ & $60-67 * *$ & $30 * *$ & $20-27 * *$ & $13-20$ & $16-19$ \\
\hline $\mathrm{Hi} / \mathrm{E}$ & $5-7$ & $13-21 * *$ & - & - & - & $7-18 * *$ & $10-12$ \\
\hline $\mathrm{Hi} / \mathrm{Gc}$ & $5-7$ & - & - & - & - & $5.4-5.9 * *$ & $2.4-3.1$ \\
\hline $\mathrm{Gc} / \mathrm{E}$ & $0.9-1.2$ & - & - & - & - & $1.3-3.1 * *$ & $3.5-4.8$ \\
\hline
\end{tabular}

aAuthorities of individual parasite and host taxa are provided in Table 3. *or Rhea americana (Linnaeus) (Rheiformes) with doubts (see Szidat 1928, Dubois 1938); **calculated from original descriptions. Abbreviations (used also in Table 2): At - anterior testis; B - body length; $\mathrm{Cb}$ - copulatory bursa; E - eggs; Fo - forebody; Ga - genital atrium; Gc - genital cone; H - hosts; Hi - hindbody; O - ovary; Os - oral sucker; Pg - proteolytic gland; $\mathrm{Ph}$ - pharynx; $\mathrm{Pt}$ - posterior testis; Vs - ventral sucker; B/E - body length/egg length; Gc/E - genital cone length/egg length; Hi/E - hindbody length/egg length; $\mathrm{Hi} / \mathrm{Fo}$ - hindbody length/forebody length; $\mathrm{Hi} / \mathrm{Gc}$ - hindbody length/genital cone length; $\mathrm{Ph} / \mathrm{Os}$ - pharynx length/oral sucker length; Vs/Os - sucker width ratio.

seen. Vitellaria composed of numerous small follicles arranged in two lateral rows, mostly distributed in caecal and extracaecal fields, from posterior end of ventral sucker, and extending caudally 861 to $1.16 \mathrm{~mm}$ from ovary.

Uterus greatly convoluted, filling body behind ovary, ascending to right of ovary and testis posterior, occupying intertesticular region, dorsally to ventral sucker and laterally to cirrus sac. Mature eggs numerous, dark brown, operculated, thick-walled, 26-29 × 19-21 $(27 \times 20)$ $(n=10)$. Excretory vesicle not seen. Excretory pore terminal.

H o s t: Taraba major (Vieillot) (chororó, great antshrike) (Passeriformes, Thamnophilidae).

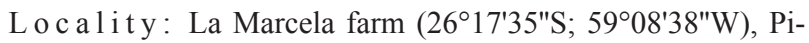
rané, Formosa Province, Argentina.

Date of collection: September 2012.

Site of infection: Bile duct.

Prevalence: $14 \%$ (1 of 7 ).

Intensity of infection: 1 .

Specimens deposited: MLP 6674.

Remarks. The morphological features of the specimen studied herein correspond to those of $L$. oswaldoi given by Travassos (1944), particularly with specimens from the grassland sparrow, Ammodramus humeralis humeralis (Bosch) [cited as Myospiza humeralis humeralis (Bosch)] (Passeriformes, Emberizidae) from Brazil characterized by having the vitellaria extending from the posterior border of the ventral sucker. Moreover, this species was reported parasitizing ardeids, cracids, icterids, odontophorids, thraupids and trogonids in Brazil, and corvids, mimids and scolopacids in USA (Travassos 1944, 1945, Denton and Byrd 1951, Peet and Ulmer 1970, Dronen and Badley 1979, Muniz-Pereira et al. 2009, Noronha et al. 2009). In Argentina, Boero et al. (1972) reported the presence of this species from the intestine of Molothrus bonariensis Gmelin (Passeriformes, Icteridae). These specimens were poorly described and figured; we suppose that they were incorrectly assigned to the genus Lyperosomum Looss, 1899 because the distribution of vitelline glands does not correspond to the generic diagnosis (follicles are not distributed in two longitudinal rows). Unfortunately, their correct identification cannot be confirmed because no specimens were deposited.

Díaz et al. (2011) reported the presence of Lyperosomum sp. in the pancreas of Larus dominicanus Lichten- 
Table 2. Comparative data for Strigea orbiculata sp. n. and related species from the Ethiopian and Australasian regions.

\begin{tabular}{|c|c|c|c|c|c|c|}
\hline Species & S. orbiculata sp. n. & S. baylisi & S. nicolli & & & S. intermedia \\
\hline Locality & Argentina & Australia & Australia & & & French Guinea \\
\hline Source & Present study & $\begin{array}{l}\text { Dubois (1938), } \\
\text { Dubois and Angel (1972) }\end{array}$ & Dubois $(1937,1938)$ & Dubois (1968) & $\begin{array}{l}\text { Dubois and Angel } \\
\text { (1972) }\end{array}$ & Dubois (1968) \\
\hline $\mathrm{H}$ & $\begin{array}{l}\text { Taraba major } \\
\text { (Vieillot) }\end{array}$ & $\begin{array}{l}\text { Threskiornis spinicollis } \\
\text { (Jameson) } \\
\text { Threskiornis molucca } \\
\text { (Cuvier) } \\
\text { Platalea flavipes Gould }\end{array}$ & $\begin{array}{l}\text { Burhinus grallarius } \\
\text { (Latham) }\end{array}$ & $\begin{array}{l}\text { Burhinus grallarius } \\
\text { (Latham) } \\
\text { Grallina cyanoleuca } \\
\text { (Latham) }\end{array}$ & $\begin{array}{l}\text { Gymnorhina tibicen } \\
\text { (Latham) }\end{array}$ & $\begin{array}{l}\text { Corvus albus } \\
\text { Müller }\end{array}$ \\
\hline B & $0.96-1.2 \mathrm{~mm}$ & $1.07-2.55 \mathrm{~mm}$ & $0.720-1.7 \mathrm{~mm}$ & $<1.7 \mathrm{~mm}$ & $1.4-1.6 \mathrm{~mm}$ & $3 \mathrm{~mm}$ \\
\hline Fo & $348-580 \times 580-752$ & $420-1110 \times 450-960$ & $360-630 \times 380-600$ & $360-630 \times 380-600$ & - & $-\times 1500$ \\
\hline $\mathrm{Hi}$ & $546-774 \times 386-638$ & $630-1440 \times 360-750$ & $360-1050 \times 300-590$ & $360-1050 \times 300-590$ & - & $-\times 1100$ \\
\hline Os & $97-121 \times 101-145$ & $90-126 \times 90-115$ & $108-162 \times 100-135$ & $108-162 \times 100-135$ & $120-160 \times 155-177$ & $200-250$ \\
\hline Vs & $145-174 \times 155-184$ & $120-170 \times 120-145$ & $150 \times 100-120$ & $140-180$ & $190-230 \times 115-230$ & 300 \\
\hline Pg & $55-83 \times 107-134$ & - & - & - & - & - \\
\hline $\mathrm{Ph}$ & $71-107 \times 41-71$ & $45-90 \times 45-75$ & $70-80 \times 50-65$ & $70-80 \times 50-65$ & $85-105 \times 75-90$ & $150 \times-$ \\
\hline $\mathrm{O}$ & $46-83 \times 102-143$ & $90-180 \times 140-270$ & $110-140 \times 200-210$ & $110-140 \times 200-210$ & - & - \\
\hline At & $110-190 \times 238-369$ & $150-225 \times 190-270$ & $250-330 \times 180-250$ & $180-250 \times 250-330^{*}$ & - & - \\
\hline $\mathrm{Pt}$ & $119-186 \times 181-381$ & $150-225 \times 180-300$ & & & - & - \\
\hline $\mathrm{Cb}$ & $143-190 \times 202-217$ & - & - & - & - & - \\
\hline $\mathrm{Ga}$ & $52-83$ & $150-235$ & $70-140$ & $70-140$ & - & - \\
\hline Gc & $95-136 \times 52-71$ & $180-270 \times 140-235$ & $135-230 \times 90-145$ & $135-230 \times 90-145$ & - & - \\
\hline $\mathrm{E}$ & $100-130 \times 71-88$ & $97-106 \times 62-76$ & $90-117 \times 58-72$ & $90-117 \times 58-72$ & $108-115 \times 65-72$ & $90 \times 50$ \\
\hline $\mathrm{Hi} / \mathrm{Fo}$ & $1.3-1.8$ & $1.11-1.75$ & $1.2-2.1$ & $1.18-2.06$ & - & - \\
\hline $\mathrm{Vs} / \mathrm{Os}$ & $1.3-1.5$ & $0.75-0.79 * *$ & $1-1.2 * *$ & $1.3-1.4^{* *}$ & $0.7-1.3 * *$ & $1.2-1.5^{* *}$ \\
\hline $\mathrm{Ph} / \mathrm{Os}$ & $0.6-1.1$ & $0.5-0.7 * *$ & $0.6-0.7 * *$ & $0.5-0.6 * *$ & $0.66-0.71 * *$ & $0.6-0.8^{* *}$ \\
\hline $\mathrm{B} / \mathrm{E}$ & $8-11$ & $10-26 * *$ & $7-17 * *$ & $15-19 * *$ & $12-15^{* *}$ & $33 * *$ \\
\hline $\mathrm{Hi} / \mathrm{E}$ & $5-7$ & $6-15 * *$ & $4-11 * *$ & $3-12 * *$ & - & - \\
\hline $\mathrm{Hi} / \mathrm{Gc}$ & $5-7$ & $4-5 * *$ & $2-5 * *$ & $3-5 * *$ & - & - \\
\hline $\mathrm{Gc} / \mathrm{E}$ & $0.9-1.2$ & $1.7-2.8 * *$ & $1.8-2.1 * *$ & $1.2-2.6 * *$ & - & - \\
\hline
\end{tabular}

*We compared the original descriptions and observed that the values of length and width were inverted by Dubois (1968) when he synonymized S. suttoni Dubois, 1937 with S. nicolli; **calculated from original descriptions. See Table 1 for abbreviations.

stein (Charadriiformes, Laridae), from Península Valdés, on the Patagonian coast of Argentina. This finding of L. oswaldoi in T. major represents a new host record, the first record in thamnophilid birds and the first valid report of this species in Argentina.

\section{DISCUSSION}

The cosmopolitan genus Strigea contains 45 nominal species (Dubois 1968, 1970, 1978, 1980, 1981, 1985, 1988, Dubois and Beverley-Burton 1971, Dubois and Angel 1972, Dubois and Macko 1972, Pearson and Dubois 1985, Lunaschi and Drago 2006, 2009, 2012). The members of this genus have complex life cycles, with four obligatory hosts (Möhl et al. 2009).

The definitive hosts are infected by trophic transmission, through the ingestion of mesocercariae/metacercariae encysted in the tissues of an intermediate host. Usually, the presence of mesocercariae in the life cycle is associated with the introduction of paratenic hosts, which increases the number of hosts in the life cycle (Niewiadomska and Pojmańska 2011). The adult forms parasitize a wide range of birds unrelated phylogenetically, whereas the mesocercariae occur in anurans and snakes and the metacercariae of 'tetracotyle' type in fishes, amphibians, snakes, birds and mammals (Lutz 1933a,b, Niewiadomska 2002).
In the Neotropical Region, the adults were found mainly in falconiform and ciconiiform birds and, to a lesser extent, caprimulgiform, gruiform, passeriform, strigiform, anseriform, charadriiform, pelecaniform and trogoniform birds (Table 3 ).

The members of Strigea show varying levels of host specificity toward their definitive hosts. Based on the hosts listed in Table 3, six species can be considered euryxenous by infecting phylogenetically unrelated hosts (S. arcuata, S. bulbosa, S. elliptica, S. falconis brasiliana, S. sphaerocephala and $S$. vaginata), and seven species as oioxenous by parasitizing a single host species ( $S$. caluri, S. caryophylla, S. inflecta, S. magniova, S. meridionalis, S. nugax and S. orbiculata sp. n.).

The spectrum of definitive hosts can be related with the similarity in their feeding habits, such is the case of $S$. arcuata reported from two species of mainly ornitophagous birds, or by having a wide spectrum of intermediate hosts, such as Strigea vaginata, the life cycle of which includes mesocercariae in frogs, e.g. Leptodactylus pentadactylus (Laurenti), Scinax ruber (Laurenti) (as Hyla ruber Daudin) and Hypsiboas crepitans (Wied-Neuwied) (as Hyla c. Wied-Neuwied) and snakes, the metacercariae in Callichthys callichthys (Linnaeus) (Siluriformes), Cerdocyon thous azarae (Wied-Neuwied) (as Canis azarae Wied-Neuwied) (Canidae) and Galictis vittata (Schre- 
Table 3. List of definitive host species for Neotropical Strigea spp.

\begin{tabular}{|c|c|c|c|c|}
\hline \multirow[t]{2}{*}{ Species } & \multicolumn{3}{|l|}{ Hosts } & \multirow[t]{2}{*}{ References } \\
\hline & Order & Family & Species & \\
\hline \multirow{2}{*}{$\begin{array}{l}\text { Strigea arcuata } \\
\text { Dubois, } 1988\end{array}$} & \multirow[t]{2}{*}{ Falconiformes } & \multirow[t]{2}{*}{ Accipitridae } & Accipiter erythronemius (Kaup) & Dubois 1988 \\
\hline & & & Parabuteo unicinctus (Temminck) & Dubois 1988 \\
\hline \multirow{4}{*}{$\begin{array}{l}\text { Strigea bulbosa } \\
\text { (Brandes, 1888) }\end{array}$} & Caprimulgiformes & Nyctibiidae & Nyctibius grandis (Gmelin) & Dubois 1968, Travassos et al. 1969 \\
\hline & \multirow[t]{2}{*}{ Ciconiiformes } & \multirow[t]{2}{*}{ Threskiornithidae } & Ajaia ajaja (Linnaeus) & Dubois 1968 , Travassos et al. 1969 \\
\hline & & & Theristicus caudatus (Boddaert) & Dubois 1968 , Travassos et al. 1969 \\
\hline & Falconiformes & Accipitridae & Elanoides forficatus (Linnaeus) & Dubois 1968, Travassos et al. 1969 \\
\hline $\begin{array}{l}\text { Strigea caluri } \\
\text { Dubois, } 1962\end{array}$ & Trogoniformes & Trogonidae & Pharomachrus mocinno De la Llave & Dubois 1968 \\
\hline $\begin{array}{l}\text { Strigea caryophylla } \\
\text { (Diesing, 1850) }\end{array}$ & Falconiformes & Accipitridae & $\begin{array}{l}\text { Accipiter bicolor pileatus } \\
\text { (Temminck) }\end{array}$ & Dubois 1968 \\
\hline \multirow{3}{*}{$\begin{array}{l}\text { Strigea elliptica } \\
\text { (Brandes, 1888) }\end{array}$} & Falconiformes & Accipitridae & Buteogallus meridionalis (Latham) & Lunaschi and Drago 2009 \\
\hline & \multirow[t]{2}{*}{ Strigiformes } & \multirow[t]{2}{*}{ Strigidae } & Bubo magellanicus (Gmelin) & Travassos et al. 1969 \\
\hline & & & Bubo virginianus nacurutu (Vieillot) & Dubois 1968 \\
\hline \multirow{12}{*}{$\begin{array}{l}\text { Strigea falconis brasiliana } \\
\text { Szidat, } 1929\end{array}$} & Charadriiformes & Laridae & Sterna sp. (experimental host) & Noronha et al. 2009 \\
\hline & \multirow[t]{2}{*}{ Ciconiiformes } & Ardeidae & Tigrisoma lineatum (Boddaert) & Noronha et al. 2009 \\
\hline & & Cathartidae & Coragyps atratus (Bechstein) & Dubois 1970 \\
\hline & \multirow[t]{7}{*}{ Falconiformes } & \multirow[t]{5}{*}{ Accipitridae } & Buteo albicaudatus Vieillot & Travassos et al. 1969 \\
\hline & & & Buteo jamaicensis umbrinus Bangs & $\begin{array}{l}\text { Pérez Vigueras 1944, 1955, } \\
\text { Dubois } 1968\end{array}$ \\
\hline & & & Buteo platypterus cubanensis Burns & Dubois and Macko 1972 \\
\hline & & & Rupornis magnirostris (Gmelin) & $\begin{array}{l}\text { Travassos et al. 1969, } \\
\text { Lunaschi and Drago } 2006\end{array}$ \\
\hline & & & Spizaetus ornatus (Daudin) & Travassos et al. 1969 \\
\hline & & \multirow[t]{2}{*}{ Falconidae } & Caracara plancus (Miller) & Dubois 1968 \\
\hline & & & Herpetotheres cachinnans (Linnaeus) & Travassos et al. 1969 \\
\hline & Gruiformes & Rallidae & Gallinula galeata (Lichtenstein) & Caballero and Díaz Ungría 1958 \\
\hline & Pelecaniformes & Phalacrocoracidae & Phalacrocorax brasilianus (Gmelin) & Drago et al. 2011 \\
\hline $\begin{array}{l}\text { Strigea inflecta } \\
\text { Lunaschi et Drago, } 2012\end{array}$ & Gruiformes & Cariamidae & Cariama cristata (Latham) & Lunaschi and Drago 2012 \\
\hline $\begin{array}{l}\text { Strigea magniova } \\
\text { Dubois, } 1988\end{array}$ & Falconiformes & Accipitridae & Rupornis magnirostris (Gmelin) & $\begin{array}{l}\text { Dubois 1988, } \\
\text { Lunaschi and Drago } 2006\end{array}$ \\
\hline $\begin{array}{l}\text { Strigea meridionalis } \\
\text { Lunaschi et Drago, } 2009\end{array}$ & Falconiformes & Accipitridae & Buteogallus meridionalis (Latham) & Lunaschi and Drago 2009 \\
\hline \multirow{2}{*}{$\begin{array}{l}\text { Strigea microbursa } \\
\text { Pearson et Dubois, } 1985\end{array}$} & \multirow[t]{2}{*}{ Falconiformes } & \multirow[t]{2}{*}{ Accipitridae } & Buteogallus meridionalis (Latham) & Lunaschi and Drago 2009 \\
\hline & & & Spilornis cheela (Latham) & Pearson and Dubois 1985 \\
\hline $\begin{array}{l}\text { Strigea nugax } \\
\text { Szidat, } 1928\end{array}$ & Ciconiiformes & Ciconiidae & Mycteria americana Linnaeus & Travassos et al. 1969 \\
\hline \multirow{4}{*}{$\begin{array}{l}\text { Strigea orbiculata } \mathbf{s p .} \mathbf{n} \text {. } \\
\text { Strigea sphaerocephala } \\
\text { (Westrumn, 1823) }\end{array}$} & Passeriformes & Thamnophilidae & Taraba major (Vieillot) & Present study \\
\hline & Anseriformes & Anatidae & Amazonetta brasiliensis (Gmelin) & Travassos et al. 1969 \\
\hline & Passeriformes & Cotingidae & Pyroderus scutatus Shaw & Dubois 1968, Travassos et al. 1969 \\
\hline & & Icteridae & Psarocolius decumanus (Pallas) & Dubois 1968, Travassos et al. 1969 \\
\hline \multirow{10}{*}{$\begin{array}{l}\text { Strigea vaginata } \\
\text { (Brandes, 1888) }\end{array}$} & Anseriformes & Anatidae & Amazonetta brasiliensis (Gmelin) & Dubois 1970 \\
\hline & \multirow[t]{6}{*}{ Ciconiiformes } & \multirow[t]{4}{*}{ Cathartidae } & Cathartes aura aura (Linnaeus) & Dubois and Macko 1972 \\
\hline & & & $\begin{array}{l}\text { Cathartes burrovianus urubitinga } \\
\text { Pelzeln }\end{array}$ & Travassos et al. 1969 \\
\hline & & & Coragyps atratus (Bechstein) & $\begin{array}{l}\text { Lutz 1928, Dubois } 1938, \\
\text { Drago and Lunaschi } 2011\end{array}$ \\
\hline & & & Sarcoramphus papa (Linnaeus) & Travassos et al. 1969 \\
\hline & & Threskiornithidae & Cercibis oxycerca (Spix) & Dubois 1981 \\
\hline & & & Theristicus caudatus (Boddaert) & Dubois 1981 \\
\hline & Falconiformes & Accipitridae & Spizaetus ornatus (Daudin) & Travassos et al. 1969 \\
\hline & & Falconidae & Caracara plancus (Miller) & Dubois 1978 \\
\hline & Gruiformes & Cariamidae & Cariama cristata (Latham) & Travassos et al. 1969 \\
\hline
\end{tabular}

ber), snakes and birds (Lutz 1933a,b, Pearson 1958). This broad range of intermediate hosts allows birds with different feeding habits to acquire this parasite. Furthermore, it allows us to confirm the doubts of Dubois $(1968,1970)$ about the report of Amazonetta brasiliensis (Gmelin) as a host of $S$. vaginata, because this anatid eats fruits, roots and some invertebrates such as insects (Carboneras 1992).
In the other euryxenous species, the intermediate hosts are unknown, but considering the feeding habits of their definitive hosts, we can assume that they also have several intermediate hosts in their life cycle.

Strigea bulbosa was reported from four species of birds with very different ecological niches, such as the threskiornithids Ajaia ajaja (Linnaeus) and Theristicus 
caudatus (Boddaert), which live in a aquatic environment, the nyctibiid, Nyctibius grandis (Gmelin) with terrestrial habits and nocturnal activity, and the accipitrid, Elanoides forficatus (Linnaeus) with terrestrial habits, but with diurnal activity.

Strigea elliptica was reported from three species of birds of prey, but two of them, Bubo magellanicus (Gmelin) and Bubo virginianus nacurutu (Vieillot) (Strigidae), have nocturnal habits, and the other, Buteogallus meridionalis (Latham) (Accipitridae), is a diurnal raptor species. Strigea falconis brasiliana has a broad spectrum of definitive hosts, including raptors, scavenging and aquatic birds.

Therefore, we can assume that these species have many intermediate hosts as has S. falconis falconis Viborg, 1795 from Holarctic and Ethiopian regions (Dubois 1968). Strigea sphaerocephala was found parasitizing passeriform birds, Pyroderus scutatus Shaw (Cotingidae) and Psarocolius decumanus Pallas (Icteridae). The first eats insects, lizards and fruit (Muir et al. 2008), and the second fruits, insects, tree-frogs (Hylidae) and bird eggs (Lopes et al. 2005, Fraga 2011).

In contrast, some molecular studies on diplostomid metacercariae in freshwater fishes revealed that some apparently generalist parasites constituted a complex of cryptic species (Locke et al. 2010). We consider that similar studies should be carried out on S. falconis brasiliana from different localities to determine if the morphometrical differences described by Lunaschi and Drago (2006) correspond to intraspecific variations or the taxon represents a complex of cryptic species.

Finally, among the species considered oioxenous, four of them were reported only from Formosa Province, Argentina, S. inflecta, S. magniova, S. meridionalis and S. orbiculata sp. n. Considering that only about $10 \%$ of Argentinean birds were studied for digeneans (Lunaschi et al. 2007), future studies will probably enlarge the host range.

Acknowledgements. The authors express their gratitude to Dr. Carlos Montoya for his help and hospitality during their stay in Formosa Province and to Luis Pagano and Ignacio Roesler (Sección Ornitología División Zoología Vertebrados, Museo de La Plata) for assistance in the collection and identification of bird hosts. Both authors are members of the Comisión de Investigaciones Científicas de la Provincia de Buenos Aires (CIC) and Universidad Nacional de La Plata (UNLP), respectively. The present study was funded by CIC (Res. N ${ }^{\circ} 2410 / 12$ ) and UNLP (11/N603).

\section{REFERENCES}

Boero J.J., Led J.E., Brandetti E. 1972: Algunos parásitos de la avifauna argentina. Analecta Vet. 4: 17-37.

Caballero E., Díaz Ungría C. 1958: Intento de un catálogo de los tremátodos digéneos registrados en territorio venezolano. Mem. Soc. Cienc. Nat. La Salle 18: 19-36.

Carboneras C. 1992: Family Anatidae (ducks, geese and swans). In: J. del Del Hoyo, A. Elliott and J. Sargatal (Eds.), Handbooks of the Birds of the World. Vol. 1. Lynx Edicions, Barcelona, pp. 528-628.

Combes C. 2001: Parasitism: The Ecology and Evolution of Intimate Interactions. University of Chicago Press, Chicago and London, $728 \mathrm{pp}$.

Denton J.F., Byrd E.E. 1951: The helminth parasites of birds, III: dicrocoeliid trematodes from North American birds. Proc. U.S. Nat. Mus. 101: 157-202.

Díaz J.I., Cremonte F., Navone G.T. 2011: Helminths of the kelp gull, Larus dominicanus, from the northern Patagonian coast. Parasitol. Res. 109: 1555-1562.

Drago F.B., Lunaschi L.I. 2011: Digenean parasites of ciconiiform birds from Argentina. Rev. Mex. Biodivers. 82: 77-83.

Drago F.B., Lunaschi L.I., Schenone M. 2011: Digenean parasites of the neotropic cormorant, Phalacrocorax brasilianus (Gmelin, 1789) (Aves: Phalacrocoracidae) from Argentina: distribution extension and new host records. Check List 7: 871-875.

Dronen N.O., Badley J.E. 1979: Helminths of shorebirds from the Texas gulfcoast. I. Digenetic trematodes from the longbilled curlew, Numenius americanus. J. Parasitol. 65: 645-649.

Dubois G. 1937: Étude de quelques strigéidés d'Australie et notes sur le genre Fibricola Dubois 1932. Ann. Parasitol. Hum. Comp. 15: 231-247.

Dubois G. 1938: Monographie des Strigeida (Trematoda). Mém. Soc. Neuchâtel. Sci. Nat. 6: 1-535.
Dubois G. 1962: Les Strigeida (Trematoda) de la Collection E. van den Broek. Bull. Soc. Neuchâtel. Sci. Nat. 85: 109-120.

Dubois G. 1968: Synopsis des Strigeidae et des Diplostomatidae (Trematoda). Mém. Soc. Neuchâtel. Sci. Nat. 10: 1-258.

Dubois G. 1970: Les Strigeata (Trematoda) de la collection A. Lutz. Mem. Inst. Oswaldo Cruz 68: 169-196.

DuвoIs G. 1978: Notes Helminthologiques. IV. Strigeidae Railliet, Diplostomidae Poirier, Proterodiplostomidae Dubois et Cyathocotylidae Poche (Trematoda). Rev. Suisse Zool. 85: 607-615.

Dubois G. 1980: Du statut de quelques Strigeata La Rue, 1926 (Trematoda). VI. Bull. Soc. Neuchâtel. Sci. Nat. 103: 63-74.

Dubois G. 1981: Du statut de quelques Strigeata La Rue, 1926 (Trematoda). VII. Bull. Soc. Neuchâtel. Sci. Nat.104: 35-46.

DuBoIs G. 1985: Quelques Strigeoidea (Trematoda) récoltés chez des oiseaux du Paraguay par la Mission Claude Weber, automne 1983, du Muséum d'Histoire naturelle de Genève. Rev. Suisse Zool. 92: 641-648.

Dubois G. 1988: Quelques Strigeoidea (Trematoda) récoltés au Paraguay par les expéditions du Muséum d'Histoire naturelle de Genève, au cours des années 1979, 1982 et 1985. Rev. Suisse Zool. 95: 521-532.

Dubois G., Angel M. 1972: Strigeata of Australian birds and mammals from the Helminthological Collection of the University of Adelaide. Trans. R. Soc. S. Aust. 96: 197-215.

Dubois G., Beverley-Burton M. 1971: Quelques Strigeata (Trematoda) d'oiseaux de Rhodesie et de Zambie. Bull. Soc. Neuchâtel. Sci. Nat. 94: 5-19.

Dubois G., Маско J. 1972: Contribution a l'étude de Strigeata La Rue, 1926 (Trematoda: Strigeida) de Cuba. Ann. Parasitol. Hum. Comp. 47: 51-75.

FRAGA R.M. 2011: Family Icteridae (New World blackbirds). In: J. del Del Hoyo, A. Elliott and D.A. Christie (Eds.), Handbooks 
of the Birds of the World. Vol. 16. Lynx Edicions, Barcelona, pp. 684-807.

Kohn A., Fernandes B.M.M. 1972: Sobre a validade das espécies pertenecentes ao género Prosthogonimus Luehe, 1899, da Coleção Helmintológica do Instituto Oswaldo Cruz. Mem. Inst. Oswaldo Cruz 70: 309-325.

Locke S.A., McLaughlin D., Marcogliese D.J. 2010: DNA barcodes show cryptic diversity and a potential physiological basis for host specificity among Diplostomoidea (Platyhelminthes: Digenea) parasitizing freshwater fishes in the St. Lawrence River, Canada. Mol. Ecol. 19: 2813-2827.

Lopes L.E., Fernandes A.M., Marini M.Â. 2005: Predation on vertebrates by Neotropical passerine birds. Lundiana 6: 57-66.

Lunaschi L.I., Cremonte F., Drago F.B. 2007: Checklist of digenean parasites of birds from Argentina. Zootaxa 1403: 1-36.

Lunaschi L.I., Drago F.B. 2006: Strigeid parasites of the roadside hawk, Buteo magnirostris (Aves: Falconiformes), from Argentina. Zootaxa 1106: 25-33.

Lunaschi L.I., Drago F.B. 2009: Species of Strigea (Digenea: Strigeidae), parasites of the savanna hawk Buteogallus meridionalis (Aves: Accipitridae) from Argentina, with the description of a new species. Folia Parasitol. 56: 268-274.

Lunaschi L.I., Drago, F.B. 2012: Digenean parasites of Cariama cristata (Aves: Gruiformes) from Formosa Province, Argentina, with the description of a new species of the genus Strigea. Acta Parasitol. 57: 26-33.

Lutz A. 1928: Estudios sobre trematodes observados en Venezue1a. Estudios de Zoología y Parasitología Venezolanas: 105-125.

Lutz A. 1933a: Considerações sobre o Distomum tetracystis Gastaldi e formas semelhantes, erroneamente chamadas Agamodistomos. Mem. Inst. Oswaldo Cruz 27: 33-49.

Lutz A. 1933b: Notas sobre dicranocercarias brazileiras. Mem. Inst. Oswaldo Cruz 27: 349-377.

Möhl K., Grosse K., Hamedy A., Wüste T., Kabelitz P., LückER E. 2009: Biology of Alaria spp. and human exposition risk to Alaria mesocercariae - a review. Parasitol. Res. 105: 1-15.

Muir J.A., Licata D., Martin T.E. 2008: Reproductive Biology of the Red-ruffed Fruitcrow (Pyroderus scutatus granadensis). Wilson J. Ornithol. 120: 862-867.

Muniz-Pereira L.C., Vieira F.M., Luque J.L. 2009: Checklist of helminth parasites of threatened vertebrate species from Brazil. Zootaxa 2123: 1-45.

Received 19 December 2012
Niewiadomska K. 2002: Family Strigeidae Railliet, 1919 In: D.I. Gibson, A. Jones and R.A. Bray (Eds.), Keys to the Trematoda. Vol. 1. CABI, Wallingford, UK, and The Natural History Museum, London, pp. 231-241.

Niewiadomska K., Pojmańska T. 2011: Multiple strategies of digenean trematodes to complete their life cycles. Wiad. Parazytol. 57: 233-241.

Noronha, D., Sá M.R., Knoff M., Muniz-Pereira L.C., Pinto R.M. 2009: Adolpho Lutz and the Helminthological Collection of the Oswaldo Cruz Institute. Ed. Museu Nacional, Rio de Janeiro, $154 \mathrm{pp}$.

Pearson J.C. 1958: Observations on the morphology and life cycle of Strigea elegans (Chandler et Rausch, 1947) (Trematoda: Strigeidae). J. Parasitol. 45: 155-174.

Pearson J.C., Dubois G. 1985: Strigeida d'Indonésie et de Malaisie, et quelques-uns d'Australie et de Tasmanie. I. Strigeoidea. Bull. Soc. Neuchâtel. Sci. Nat. 108: 5-21.

Peet S., Ulmer M.J. 1970: Trematode parasites of the brown thrasher, Toxostoma rufum, from Dickinson County, Iowa. Proc. Iowa Acad. Sci. 77: 196-199.

Pérez Vigueras I. 1944: Trematodes de la super-familia Strigeoidea; descripción de un género y siete especies nuevas. Rev. Univ. La Habana 52-54: 294-314.

Pérez Vigueras I. 1955: Contribución al conocimiento de la fauna helmintológica cubana. Mem. Soc. Cub. Hist. Nat. 22: 195-232.

SzIDAT L. 1928: Zur Revision der Trematodengattung Strigea Abildgaard. Zbl. Bakteriol. 1. Abt., Orig. 105: 204-215.

Travassos L. 1944: Revisão da Familia Dicrocoeliidae Odhner, 1910. Monografia do Instituto Oswaldo Cruz, Rio de Janeiro, No. 2, 357 pp.

Travassos L. 1945. Notas sobre Dicrocoeliidae. Mem. Inst. Oswaldo Cruz 42: 629-633.

Travassos L., Freitas J.F.K., Kohn A. 1969: Trematódeos do Brasil. Mem. Inst. Oswaldo Cruz 67: 1-886.

Vicente J., Magalhães Pinto R., Noronha, D. 1982: Estudo das especies brasileiras do genero Diplotriaena Henry et Ozoux, 1909 (Nematoda, Filarioidea). Mem. Inst. Oswaldo Cruz 78: 165-182.

Zimmer K.J., IsLer M.L. 2003: Family Thamnophilidae (typical antbirds). In: J. del Hoyo, A. Elliot and D.A. Christie (Eds.), Handbook of the Birds of the World. Vol. 8. Lynx Editions, Barcelona, pp. $448-681$.

Accepted 29 April 2013 